\title{
The Influence of Culture Age on the Cellular Fatty Acid Composition of Four Selected Yeasts
}

\author{
By BENNIE C. VILJOEN, * JOHAN L. F. KOCK \\ AND PIETER M. LATEGAN \\ Department of Microbiology, University of the Orange Free State, PO Box 339, \\ Bloemfontein 9300, South Africa
}

(Received 26 November 1985; revised 17 February 1986)

\begin{abstract}
Cellular fatty acids were extracted from cells of Debaryomyces vanrijiae, Saccharomycodes ludwigii, Endomyces fibuliger and Metchnikowia reukaufii at different growth stages of batch growth and analysed by gas chromatography. A high degree of variation in relative concentrations, as well as in composition, of fatty acids, was observed during the exponential and early stationary growth phases. Greater reproducibility and a relatively stable fatty acid composition were obtained with cells harvested in the late stationary phase.
\end{abstract}

\section{INTRODUCTION}

It has been proposed that the cellular fatty acid composition of bacteria (Athalye et al., 1984; Moss, 1981; Shaw, 1974) and yeasts (Gangopadhyay et al., 1979; Kaneko et al., 1976; Moss et $a l ., 1982)$ can be used for identification purposes. It has been widely reported that the fatty acid composition of micro-organisms may vary with culture conditions and age (Choi et al., 1982; Dawson \& Craig, 1965; Hunter \& Rose, 1971; Johnson et al., 1972; Marr \& Ingraham, 1962; McMurrough \& Rose, 1971; Uzuka et al., 1985). Direct comparisons between the fatty acids of yeasts in the different investigations cannot, therefore, be made effectively.

In this study, the influence of culture age on the fatty acid composition of four yeasts representing four families was investigated. The optimum culture age resulting in the most reproducible fatty acid composition was identified in order to construct a reliable identification process for yeasts.

\section{METHODS}

Strains studied. Cultures were supplied by Professor J. P. van der Walt of the CSIR, Pretoria, South Africa. The following strains were investigated: Debaryomyces vanrijiae CSIR-Y960; Endomyces fibuliger CSIR-Y643; Metchnikowia reukaufi CSIR-Y13; and Saccharomycodes ludwigii CSIR-Y22.

Cultivation of strains. Stock cultures were maintained on yeast extract-malt extract (YM) slants (Wickerham, 1951). One litre conical flasks were inoculated from slants and cultured for $16 \mathrm{~h}$ at $30^{\circ} \mathrm{C}$ on a rotary shaker at

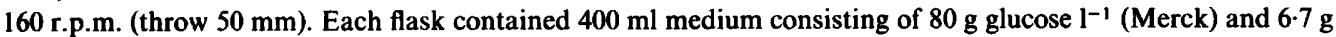
yeast/nitrogen base $\mathrm{l}^{-1}$ (YNB) (Wickerham, 1951). Samples $(18 \mathrm{ml})$ of each culture were used to inoculate each of 20 1-litre conical flasks containing the same medium. The inoculated flasks were incubated as before. Duplicate flasks of each strain were collected at the sampling times indicated. Growth was monitored by measuring the optical density of each flask at $640 \mathrm{~nm}$ with a Klett-Summerson colorimeter. All growth experiments were done in triplicate for each yeast. After the appropriate time intervals, cells were harvested by centrifugation at $8000 \mathrm{~g}$ for 5 min at $4{ }^{\circ} \mathrm{C}$, washed and recentrifuged three times, and resuspended in cold $0.85 \% \mathrm{NaCl}$. The cells were then stored at $-15^{\circ} \mathrm{C}$.

Fatty acid analysis. The extraction and preparation of fatty acid methyl esters were done according to the method of Gunasekaran \& Hughes (1980). The final extraction of the fatty acid methyl esters was done using nhexane. The fatty acid methyl esters were analysed by GC on a Hewlett-Packard model 5830A gas chromatograph equipped with dual flame-ionization detectors. 


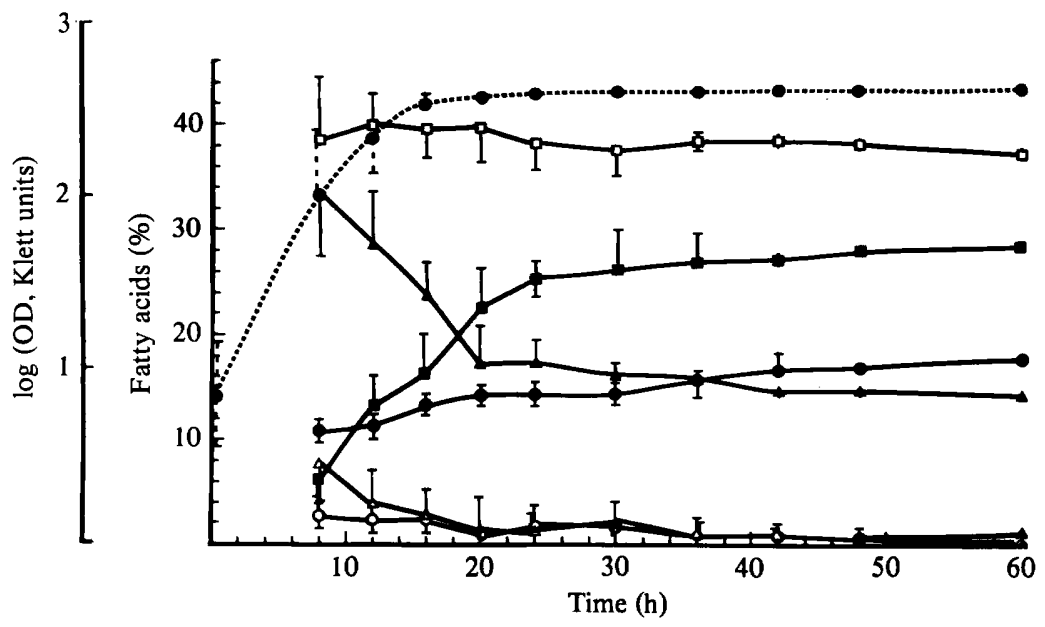

Fig. 1. Changes in fatty acid composition in Debaryomyces vanrijiae (Y960). Each point represents the mean of triplicate cultures., Palmitic acid; $O$, palmitoleic acid; $\square$, stearic acid; $\square$, oleic acid; $\boldsymbol{\Lambda}$, linoleic acid; $\triangle$, linolenic acid; $\cdots \cdot$, optical density.

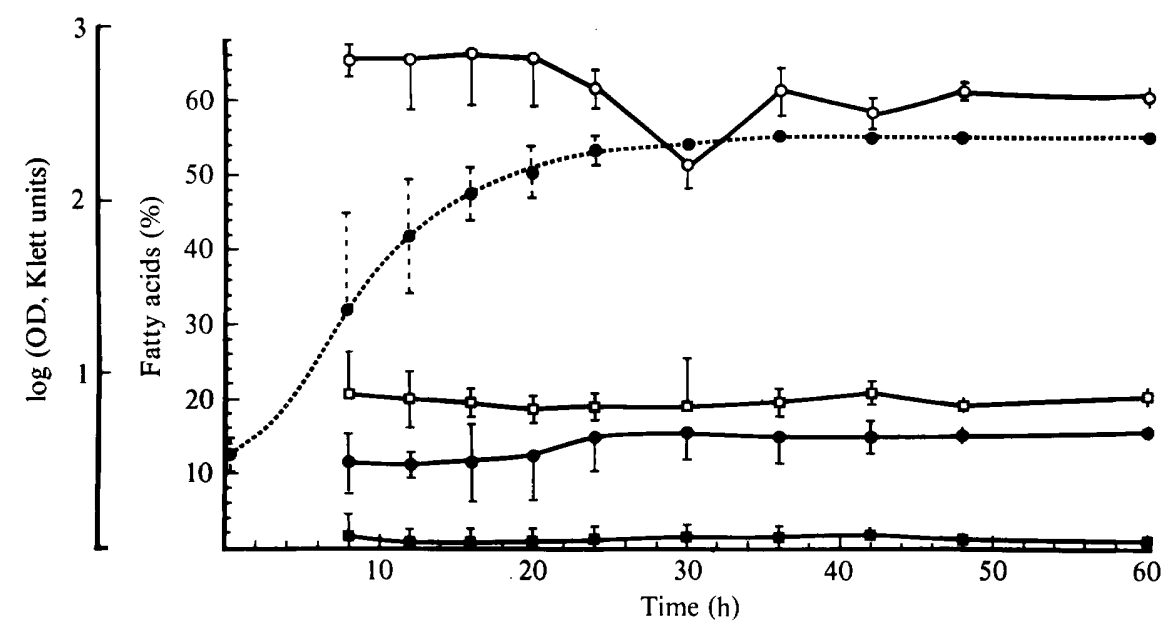

Fig. 2. Changes in fatty acid composition in Saccharomycodes ludwigii (Y22). Reproducibility of results and symbols are as in Fig. 1.

Identification of the esters was based on the comparison of retention times with known standards of C14:0 (myristic acid), C14:1 (myristoleic acid) C16:0 (palmitic acid), C16:1 (palmitoleic acid), C18:0 (stearic acid), $\mathrm{C} 18: 1$ (oleic acid), $\mathrm{C} 18: 2$ (linoleic acid) and $\mathrm{C} 18: 3$ (linolenic acid) (Serva). Analyses were done on glass columns $(4 \mathrm{~mm}$ i.d. $\times 1.5 \mathrm{~m})$ packed with $5 \%$ diethyleneglycol succinate on Chromosorb W (80-100 mesh; Anatech). The flow rate of the nitrogen carrier gas was $40 \mathrm{ml} \mathrm{min}^{-1}$ at a column temperature of $160^{\circ} \mathrm{C}$.

\section{RESULTS AND DISCUSSION}

The changes in optical density and fatty acid composition during incubation of the different strains are shown in Figs 1-4. Each point represents the mean of three repetitions and the bars indicate SD. The fatty acids of the four strains were predominantly esters of palmitic $(16: 0)$, palmitoleic $(16: 1)$, stearic $(18: 0)$, oleic $(18: 1)$, linoleic $(18: 2)$ and linolenic $(18: 3)$ acids in various proportions. Since myristic $(14: 0)$ and myristoleic acid $(14: 1)$ never accounted for more than $2 \%$ of the total fatty acid content, the results for these two fatty acids were omitted. 


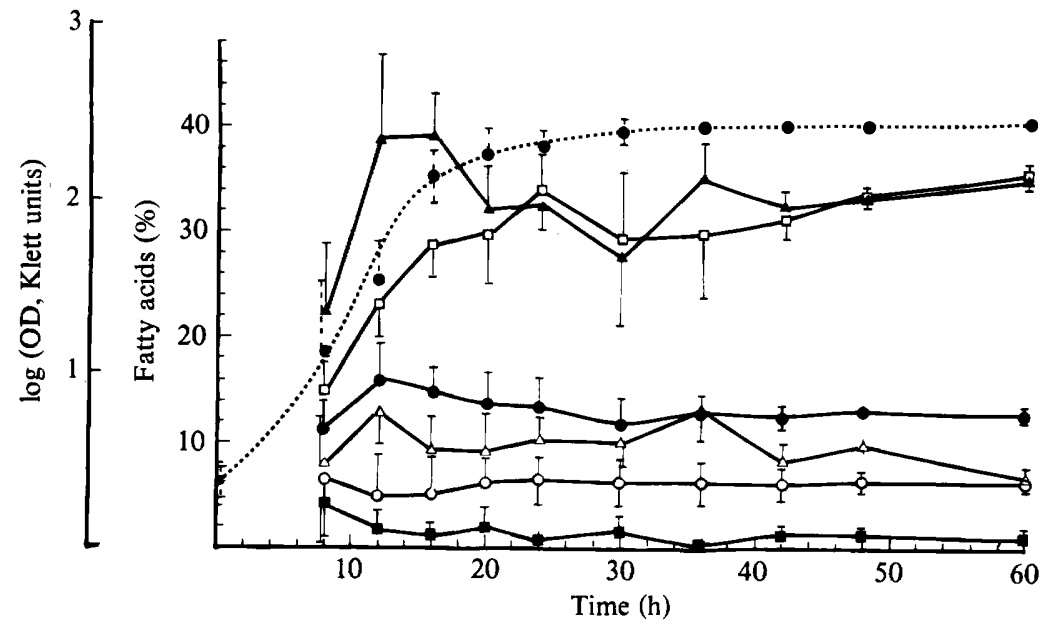

Fig. 3. Changes in fatty acid composition in Metchnikowia reukaufi (Y13). Reproducibility of results and symbols are as in Fig. 1.

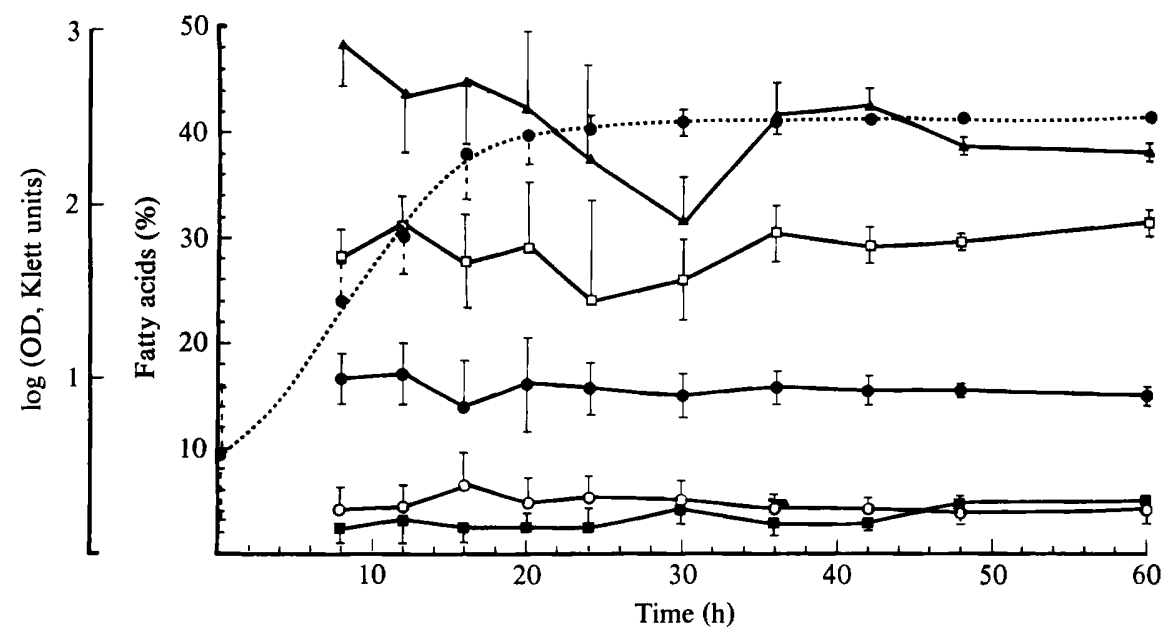

Fig. 4. Changes in fatty acid composition in Endomyces fibuliger (Y643). Reproducibility of results and symbols are as in Fig. 1 .

Significant changes in fatty acid composition of the four strains studied occurred only during the exponential and early stationary phases $(8-24 \mathrm{~h}$ ) (Figs 1-4). During the late stationary phase $(24-60 \mathrm{~h})$ the different fatty acids studied remained constant except in $S$. ludwigii, where a significant decrease in palmitoleic acid $(16: 1)$ was observed between 20 and $30 \mathrm{~h}$, followed by an increase to a level slightly lower than the original (Fig. 2).

An increase in stearic acid $(18: 0)$ occurred up to $24 \mathrm{~h}$ in D. vanrijiae (Fig. 1), while palmitic acid $(16: 0)$ increased steadily. Palmitoleic acid $(16: 1)$, linolenic acid $(18: 3)$ and linoleic acid $(18: 2)$ decreased during the exponential phase in this yeast. The other fatty acids studied remained constant during the entire growth cycle.

Oleic acid (18:1) and linoleic acid (18:2) increased significantly in $M$. reukaufi during the exponential phase (Fig. 3). Culture age had no significant effect on the relative amounts of the other fatty acids present in this organism. No significant change was observed in the long-chain fatty acid composition of $E$. fibuliger up to $60 \mathrm{~h}$ (Fig. 4).

Variations in culture optical density coincided with those observed for fatty acids (Figs 1-4). 
This indicates that replicate cultures were in different stages of growth when harvested at a given time, resulting in the variations in fatty acid composition observed. Based on these results, a culture age of $48 \mathrm{~h}$ was selected as optimal for the analysis of fatty acids for identification purposes. This study should be extended to include more yeasts in order to establish the general validity of the proposed standard technique.

The authors are indebted to P. J. Botes for gas-chromatographic assistance and Carol Viljoen for excellent secretarial work.

\section{REFERENCES}

Athalye, M., Noble, W. C. \& Minnikin, D. E. (1984). Analysis of cellular fatty acids by gas chromatography as a tool in the identification of medically important coryneform bacteria. Journal of Applied Bacteriology 58, 507-512.

Chol, S. Y., Ryu, D. D. Y. \& RheE, J. S. (1982). Production of microbial lipid: effects of growth rate and oxygen on lipid synthesis and fatty acid composition of Rhodotorula gracilis. Biotechnology and Bioengineering 24, 1165-1172.

Dawson, P. S. S. \& CraIG, B. M. (1965). Lipids of Candida utilis: changes with growth. Canadian Journal of Microbiology 12, 775-785.

Gangopadhyay, P. K., Thadepalli, H., Roy, I. \& ANSARI, A. (1979). Identification of species of Candida, Cryptococcus and Torulopsis by gas-liquid chromatography. Journal of Infectious Diseases 140, 952-958.

Gunasekaran, M. \& Hughes, W. T. (1980). Gasliquid chromatography: a rapid method for identification of different species of Candida. Mycologia 72, 505-511.

Hunter, K. \& Rose, A. H. (1971). Lipid composition of Saccharomyces cerevisiae as influenced by growth temperature. Biochimica et biophysica acta 260, 639653.

Johnson, B., Nelson, S. J. \& Brown, C. M. (1972). Influence of glucose concentration on the physiology and lipid composition of some yeasts. Antonie van Leeuwenhoek 38, 129-136.
Kaneko, H., Hosohara, M., Tanaka, M. \& ITOH, T. (1976). Lipid composition of 30 species of yeast. Lipids 11, 837-844.

McMurrough, I. \& Rose, A. H. (1971). Effects of temperature variation on the fatty acid composition of Candida utilis. Journal of Bacteriology 107, 753758.

MARR, A. G. \& INGRAHAM, J. L. (1962). Effect of temperature on the composition of fatty acids in Escherichia coli. Journal of Bacteriology 84, 12601267.

Moss, C. W. (1981). Gas-liquid chromatography as an analytical tool in microbiology. Journal of Chromatography 203, 337-347.

Moss, C. W., Shinoda, T. \& Samuels, J. W. (1982). Determination of cellular fatty acid compositions of various yeasts by gas-liquid chromatography. Journal of Clinical Microbiology 16, 1073-1079.

SHaw, N. (1974). Lipid composition as a guide to the classification of bacteria. Advances in Applied Microbiology 17, 63-108.

UzuKa, Y., Naganuma, T., Tanaka, K. \& Suzuki, K. (1985). Relation between neutral lipid accumulation and the growth phase in the yeast, Lipomyces starkeyi, a fat producing yeast. Agricultural and Biological Chemistry 49, 851-852.

WICKERHAM, L. J. (1951). Taxonomy of yeasts. Technical bulletin no. 1029, United States Department of Agriculture. 